\title{
A Case of Tophaceous Gout in the Lumbar Spine: A Review of the Literature and Treatment Recommendations
}

\author{
Mithulan Jegapragasan ${ }^{1}$ Alejandro Calniquer ${ }^{1}$ William D. Hwang ${ }^{2}$ Quynh T. Nguyen ${ }^{2}$ Zachary Child ${ }^{1}$ \\ ${ }^{1}$ Department of Orthopaedics and Sports Medicine, University of \\ Address for correspondence Mithulan Jegapragasan, MD, \\ Washington, Seattle, United States \\ 2 Department of Radiology, University of Washington, Seattle, \\ United States \\ Department of Orthopaedics and Sports Medicine, University of \\ Washington, 1959 NE Pacific Street, Box 356500, Seattle, WA 98195- \\ 650, United States (e-mail: mithulan@uw.edu).
}

Evid Based Spine Care J 2014;5:52-56.

\begin{abstract}
Study Design Case report.

Objective The objective of this study is to report the occurrence of tophaceous gout in the lumbar spine.

Methods Using a case report to illustrate the key points of gout in the spine, we provide a brief review of gout in the literature as it relates to its orthopedic and spinal manifestations as well as guidelines for management.

Results This case report details the occurrence of a large and clinically significant finding of tophaceous gout in the lumbar spine in a 24-year-old man with a known history of gout and a 3-year history of progressive back pain.

\section{Keywords}

- gout

- hyperuricemia

- radiculopathy

- lower back pain

Conclusion A high index of suspicion can assist in diagnosis of patients presenting with back pain or neurologic findings with a history of gout. A previous history of gout (especially the presence of tophi), hyperuricemia, and the radiological characteristics presented here should aid the clinician in making the diagnosis of spinal gout. Early diagnosis has the potential to prevent the need for surgical intervention.
\end{abstract}

\section{Introduction}

Gout is a common inflammatory arthritis that is characterized by the deposition of monosodium urate crystals in the synovium. An estimated 6.1 million adults in the United States have gout. ${ }^{1}$ Monosodium urate crystals have decreased solubility at lower temperatures, and consequently, the most commonly affected sites are peripheral joints. Other crystalline arthropathies include pseudogout. Pseudogout is the deposition of calcium pyrophosphate dihydrate crystals in the joints or periarticular structures, which leads to inflammation in joints. Pseudogout shows similar clinical findings to gouty arthritis although tophi are exclusive to gout.

Occurrence of gout in the spine is rare, with little over 70 cases reported in the literature. Not all levels of the spine are equally likely to be affected. One review of the literature found that the lumbar spine was most commonly affected $(56 \%$ of cases), followed by the cervical and thoracic spine, which both occurred in $22 \%$ of cases. ${ }^{2}$ Out of these cases, the most common presenting symptoms were nonspecific paraparesis (39.0\%), radiculopathy $(27.0 \%)$, and back pain $(18.0 \%){ }^{3}$ Given the rare incidence and nonspecific presentation, an inclusion of gout in the differential diagnosis of atypical neurocompressive pathologies is necessary for diagnosis. In the spine, gout may affect the epidural space, ligamentum flavum, intervertebral disc, pedicles, facet joint capsule, and neural foramen. ${ }^{4}$ This may present as spinal stenosis, lumbar radiculopathy, spondylolisthesis, or cauda equina syndrome.

Biopsy is the gold standard in confirming the diagnosis. Histologic findings show a granulomatous infiltrate of received

July 9,2013

accepted

December 2, 2013 (c) 2014 Georg Thieme Verlag KG Stuttgart · New York
DOI http://dx.doi.org/ 10.1055/s-0034-1366979. ISSN 1663-7976. 


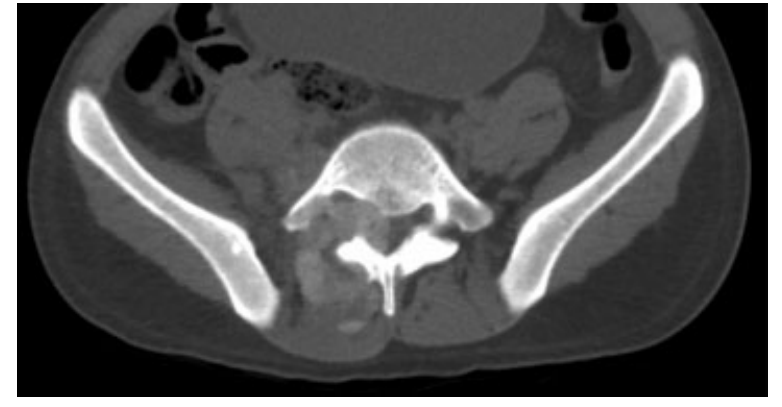

Fig. 1 Axial noncontrast CT at the L5-S1 level shows a hyperattenuating, partially calcified soft tissue mass scalloping the posterior vertebral body, widening the right neural foramen, and eroding the right facet joint. CT, computed tomography.

multinucleated giant cells, histiocytes, and fibroblasts. In addition, specimens examined under polarized light microscopy demonstrate the characteristic needle-shaped crystals with negative birefringence of gout. High serum urate levels, a concomitant history of advanced renal disease, previous gout attacks (podagra), and the cutaneous manifestation of tophi all point to the diagnosis.

\section{Report of a Case}

A 24-year-old man with a 4-year history of tophaceous gout and chronic kidney disease presented with a 3-year history of lower back pain that acutely worsened over the course of a week before hospital admission. He had developed shooting pain down the lateral aspect of his thigh with the right worse than the left. Of note, the patient had not been taking any of his gout medication for the six months before admission. He has had several acute flares of gout since his diagnosis and has disabling tophi in his hands.

Physical exam was notable for weakness of the right lower extremity, with 4/5 strength in ankle dorsiflexion, extension of the big toe, and ankle plantarflexion. His creatinine on admission was $4.80 \mathrm{mg} / \mathrm{dL}$ and serum uric acid was $14.6 \mathrm{mg} / \mathrm{dL}$.

Imaging initially obtained for abdominal pain and fever was suspicious for tumor (-Fig. 1), demonstrating a large, heterogeneous, infiltrating calcified soft tissue mass in the lumbosacral region, expanding the neural foramen, and scalloping the vertebral body, with erosion of the facet joints. Bone scintigraphy showed mild increased uptake in the right L4-S1 region. Magnetic resonance imaging (MRI) revealed an isointense mass surrounding the L5 nerve root on T1-weighted images (-Fig. 2), with extensive cystic component on T2weighted images (-Fig. 3). Postcontrast sequences were not performed due to poor renal function. Tissue diagnosis was then indicated although the concordance of imaging with the patient's history and physical exam suggested gout. The patient underwent ultrasound-guided fluid aspiration, which revealed negatively birefringent crystals on polarized light microscopy, confirming the diagnosis of gout (-Fig. 4).

The patient was taken to the operating room for lumbar decompression. Intraoperatively, there was significant mar-

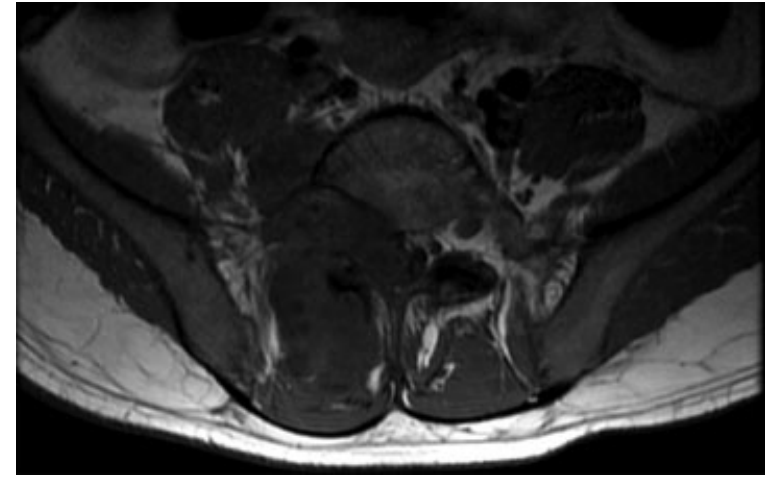

Fig. 2 Axial T1-weighted MRI at the L5-S1 level demonstrates a heterogeneous soft tissue mass mostly isointense to muscle surrounding the exiting right $\mathrm{L} 5$ nerve root and extending anteriorly into the retroperitoneum, with an epidural component causing dural compression and displacement. MRI, magnetic resonance imaging.

ginal sclerosis in areas of the sacrum, especially the right sacral ala and S1 foramen. The mass had also grown into the paraspinal muscles and into the retroperitoneum along the L5 nerve root. A decompressive laminectomy of L4-S1 and resection of intraspinal canal lesion and perineural lesion was performed (-Fig. 5 ). The right $\mathrm{L} 4$ pedicle extending to the vertebral body was completely destroyed. Large aggregates of chalky tophi and a cystic collection of milky white fluid were intralesionally resected. Postoperatively, the patient was restarted on colchicine and allopurinol and given a brief burst of prednisone. By the 6-week clinic visit, he had regained his

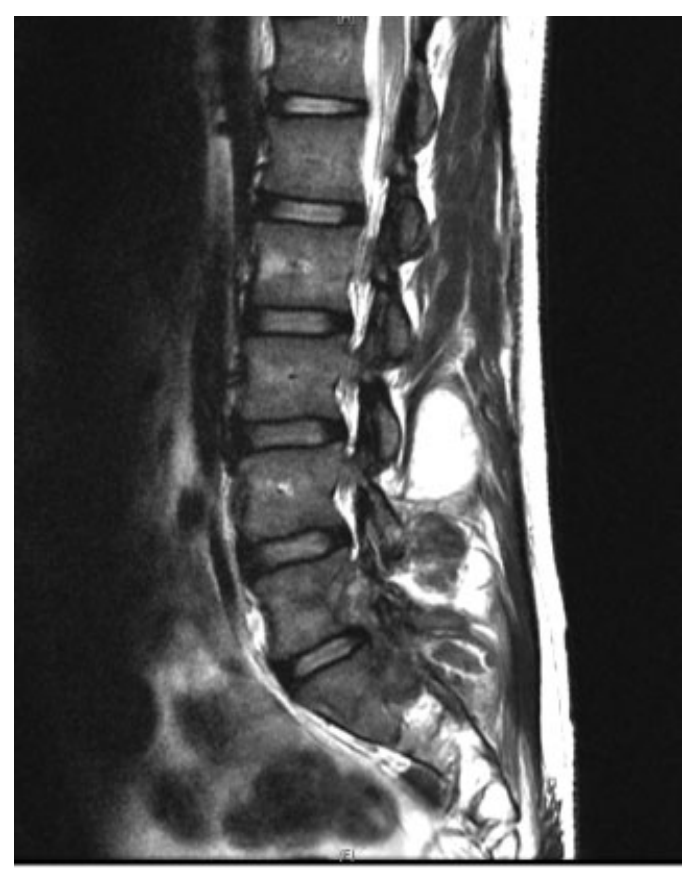

Fig. 3 Sagittal T2-weighted MRI scan shows the heterogeneous mass with soft tissue and cystic components. The solid components fill the right L5-S1 and S1-2 neural foramina, with scalloping of the vertebral bodies. There is facet joint erosive change at both L4-5 and L5-S1. MRI, magnetic resonance imaging. 


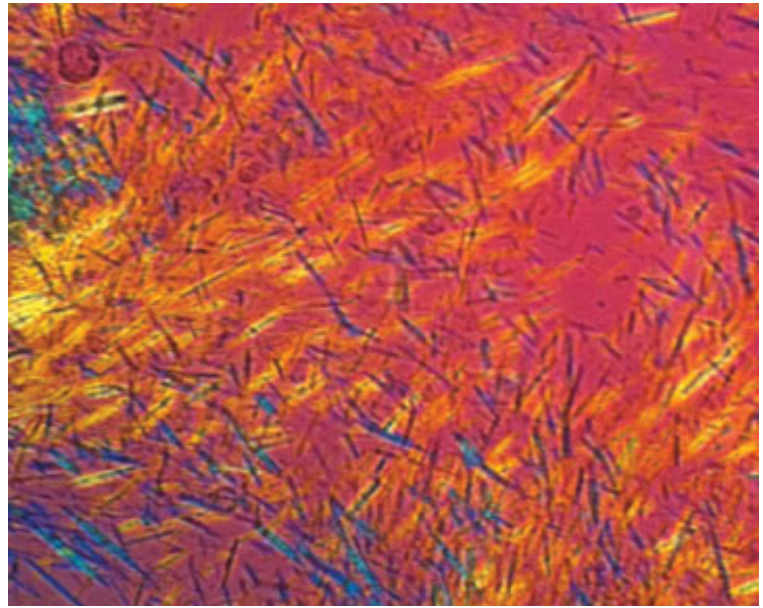

Fig. 4 Polarized light microscopy of ultrasound-guided fluid aspirate of paraspinal mass reveals numerous needle-like negatively birefringent monosodium urate crystals.

strength in his right lower extremity and had interval resolution of his lower back pain.

\section{Discussion}

This case describes the uncommon presentation of tophaceous deposits in the spine. As mentioned, gout rarely affects the spine and even more rarely evolves in such an aggressive fashion. Initially, it was felt that imaging and clinical symptoms favored malignancy given the progressive neurologic deficit and imaging characteristics. Physical exam and plain films revealed the presence of multiple gouty tophi in other small joints, which increased suspicion for gouty arthropathy of the spine (-Fig. 6). In the absence of such remarkable physical findings, isolated spinal gout can be extremely difficult to diagnose and as such a tissue diagnosis before intervention is highly encouraged except in the most urgent/ emergent scenarios.

Although crystal deposition disease of the spine is rare, the diagnosis can be made by the patient's history, the results of

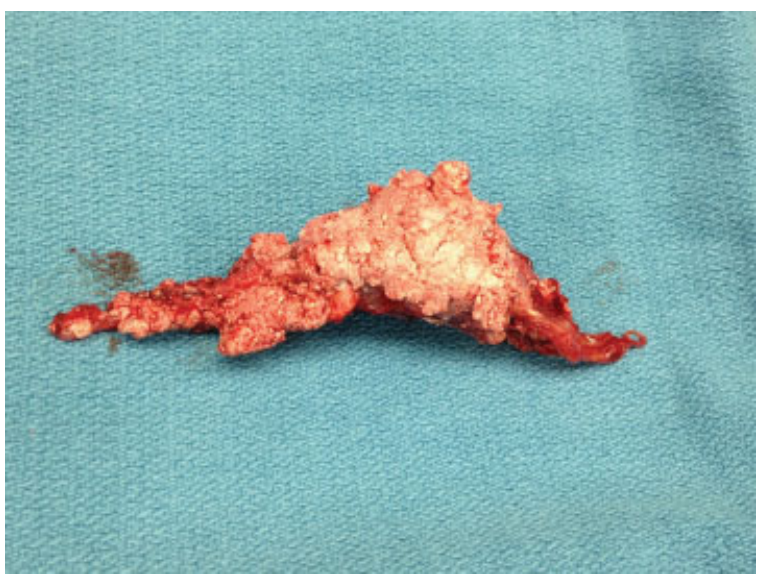

Fig. 5 Surgical pathology specimen: en bloc resection of a nodular, chalky soft tissue mass.

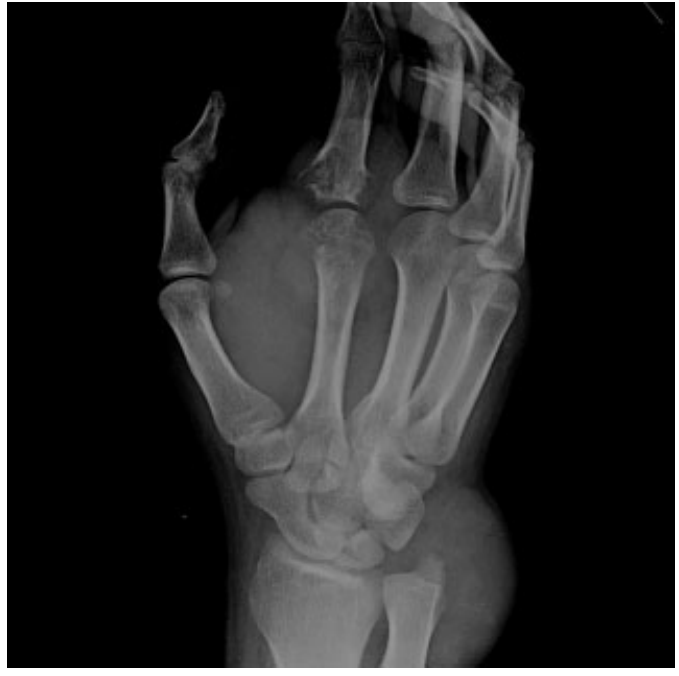

Fig. 6 Oblique radiograph of the right wrist obtained 3 years prior shows classic features of gout, including juxta-articular "punched out" erosions without osteoporosis at the second metacarpophalangeal joint and large calcific soft tissue tophus at the ulnar side of the wrist.

computed tomography (CT) or MRI of the spine, and tissue samples. Gouty tophi normally appear as a hypointense, homogenous mass associated with a joint on T1- and T2weighted MR images, which enhances with gadolinium because of vascularized reactive tissue in the tophus. ${ }^{5,6}$ Imaging characteristics of spinal gout are nonspecific, however, and the use of an image-guided fine needle aspiration or biopsy is recommended. The imaging characteristics in our case were somewhat unique given the heterogeneity of the mass demonstrating discrete areas of soft tissue, calcification, and cyst formation, raising the initial concern of a nerve sheath tumor with possible malignant degeneration. The mass was noted to extend along the right-sided L5 and S1 nerve roots. Also, we believe this massive spinal tophus to be the largest reported, measuring $7.5 \mathrm{~cm} \times 2.8 \mathrm{~cm} \times 10.3 \mathrm{~cm}$, and extending from the level of L3 to S1 in the sagittal plane. In most cases, diagnosis can only be confirmed by histological examination of biopsy material. This minimally invasive diagnostic test allows differentiation from other important imitators such as neoplastic disease or infection and avoids unnecessary exploration. Conversely obtaining a tissue diagnosis before formal intervention can avoid "unintended" intralesional resection of sarcoma. Dual-energy CT may have a future role in differentiating spinal gout from infection or tumor and obviating biopsy by identifying the presence of uric acid in tissues. Although some controversy exists, most presentations of axial pain caused by spinal gout can be medically managed. ${ }^{3}$

Patients without concomitant neurological deficits can be treated medically after spinal infection is excluded. Medical treatment with nonsteroidal anti-inflammatory agents, such as naproxen and ibuprofen, and steroids, such as prednisolone, are effective to control pain acutely. This is followed by administration of a urate-reducing agent for long-term control such as probenecid, allopurinol, or rasburicase. Maintaining adequate hydration and alkalization of urine is 
required to prevent recurrent episodes. ${ }^{3,5}$ It is important for the clinician to realize that surgery is only warranted for relief of neurologic symptoms and that long-term management hinges on adequate medical therapy. Given the long duration of this patient's axial skeletal pain, aggressive medical management may have rendered surgery unnecessary; however, a cautious approach is warranted when neurological deficits are present.

\section{Disclosures}

None

\section{Commentary}

\author{
Daniel Wendling ${ }^{1}$ \\ ${ }^{1}$ Department of Rheumatology, CHRU (University Teaching Hospital), \\ and Université de Franche-Comté, Besançon, France
}

Spine is not the most characteristic location of gout, and this is described mainly in case reports. However, this event raises diagnosis and treatment problems.

Jegapragasan et al report a case of tophaceous gout of the lumbar spine, revealed by low back pain and neurological symptoms in a patient with long-standing peripheral tophaceous gout, elevated uric acid levels, and renal impairment, in a context of treatment discontinuation. Imaging revealed a tophaceous mass surrounding zygapophysial joint, and diagnosis was confirmed by needle aspiration, revealing negatively birefringent crystals. Surgery (decompressive laminectomy and resection of intraspinal and perineural lesion) was required in this case, with favorable outcome.

The frequency of spine involvement of gout is not clearly established. In a retrospective study of patients with peripheral gout, a spine CT was available in 64 patients. Changes suggestive of gout (tophi or discovertebral erosion) were present in $14 \%$ of the cases. ${ }^{1}$ In a prospective study of 45 patients with at least 3 years duration of poorly controlled peripheral gout, spinal CT evaluation revealed lesions in 35\%, 2 a and only half of them had back pain. Every segment of the spine may be involved, but as recalled by Jegapragasan et al, the lumbar segment is the most frequent location. Different features may be seen: vertebral bone tophi, spondylodiskitis, zygapophysial tophi or destructive arthritis, and epidural mass. $^{3}$ This explains the variety of clinical symptoms (isolated back pain, neurological symptoms associated) and differential diagnosis (infection, tumoral disease, degenerative disease). In one study, ${ }^{2}$ no differences in age, gout duration, body mass index, renal function, or presence of back pain or hypertension were found between gouty patients with and without spinal lesions, but peripheric radiographic erosions and diabetes were associated with the presence of spine CT abnormalities. The presence of subcutaneous tophi and history of previous

\section{References}

1 Neogi T. Clinical practice. Gout. N Engl J Med 2011;364(5): 443-452

2 Hou LC, Hsu AR, Veeravagu A, Boakye M. Spinal gout in a renal transplant patient: a case report and literature review. Surg Neurol 2007;67(1):65-73, discussion 73

3 King JCJ, Nicholas C. Gouty arthropathy of the lumbar spine: a case report and review of the literature. Spine (Phila Pa 1976) 1997; 22(19):2309-2312

4 Hasturk AE, Basmaci M, Canbay S, Vural C, Erten F. Spinal gout tophus: a very rare cause of radiculopathy. Eur Spine J 2012;21 (Suppl 4):S400-S403

5 Chang I-C. Surgical versus pharmacologic treatment of intraspinal gout. Clin Orthop Relat Res 2005;(433):106-110

6 Kelly J, Lim C, Kamel M, Keohane C, O'Sullivan M. Topacheous gout as a rare cause of spinal stenosis in the lumbar region. Case report. J Neurosurg Spine 2005;2(2):215-217

gouty attacks are important anamnestic features that may help diagnosis, but nevertheless, gout may be revealed by spinal involvement. ${ }^{3}$ Awareness may help interpretation of imaging (plain radiography, CT, MRI). Dual-energy CT may allow in the future a most accurate imaging diagnosis approach of gout by estimates of tophus attenuation. In difficult cases, tissue samples examination remains the gold standard. Needle aspiration is possible for zygapophyseal or discal involvement; otherwise, a guided biopsy is required. However, in all the cases, polarized light microscopy examination is mandatory to establish the diagnosis.

Management of these patients may vary according to the clinical presentation. In case of neurological symptoms due to tophaceous mass, surgical decompression is needed, like in the case of Jegapragasan et al. This may also allow to confirm, or sometimes reveal ${ }^{3}$ the diagnosis. Evidence of spine location of gout requires medical treatment of gout to avoid subsequent potential neurological complications, since old (colchicine and allopurinol) and new therapeutic possibilities are available for treating inflammatory attacks (anti IL-1 strategies) and for urate lowering (febuxostat, rasburicase, and pegloticase). ${ }^{4,5}$

\section{References}

1 Konatalapalli RM, Demarco PJ, Jelinek JS, et al. Gout in the axial skeleton. J Rheumatol 2009;36(3):609-613

2 Konatalapalli RM, Lumezanu E, Jelinek JS, Murphey MD, Wang H, Weinstein A. Correlates of axial gout: a cross-sectional study. J Rheumatol 2012;39(7):1445-1449

3 Wendling D, Prati C, Hoen B, et al. When gout involves the spine: five patients including two inaugural cases. Joint Bone Spine 2013; 80(6):656-659

4 Verhoeven F, Prati C, Godfrin-Valnet M, Guillot X, Wendling D. IL1 blockade in crystal-induced arthritis: impact of disease duration and the inflammatory syndrome. Comments on the article by Couderc M, et al. "Efficacy of anakinra in articular chondrocalcinosis". Joint Bone Spine 2013;80(1):115-116

5 So A, Busso N. Update on gout 2012. Joint Bone Spine 2012;79(6): 539-543 


\section{Editorial Perspective}

For a variety of reasons, case reports are located near the bottom of the evidence pyramid. That said, they maintain a firm role in our peer-reviewed literature for the value they can provide us when they highlight rarities and raise our awareness for unusual cases that we as clinicians may be exposed to on any given day. The topic of tophaceous gout affecting the spine is such an unusual case; it fascinates with its variability of presentation and clinical course and, therefore, lacks clear diagnostic and treatment guidelines. This well-documented case and thoughtful commentary impresses upon us the importance of a thorough physical examination of spine patients, including integument and joints outside the axial skeleton. It also underscores the importance of being familiar with differential diagnoses as we look at less than straightforward cases. It stands to reason that an earlier diagnosis in this patient would very reasonably have allowed for nonsurgical medical treatment and helped avoid surgery and subsequent disability. 